\section{REFERENCES}

Bendifallah S, Uzan C, Fauvet R, Morice P, Darai E (2013) External multicentre validation of a nomogram predicting the risk of relapse in patients with borderline ovarian tumours. $\mathrm{Br} J$ Cancer 109: 2774-2777.

Iasonos A, Schrag D, Raj GV, Panageas KS (2008) How to build and interpret a nomogram for cancer prognosis. J Clin Oncol 26: 1364-1370.
Obermair A (2014) Comment on 'External multicentre validation of a nomogram predicting the risk of relapse in patients with borderline ovarian tumours'. Br J Cancer 111: 2375.

Obermair A, Tang A, Kondalsamy-Chennakesavan S, Ngan H, Zusterzeel P, Quinn M, Carter J, Leung Y, Janda M (2013) Nomogram to predict the probability of relapse in patients diagnosed with borderline ovarian tumors. Int J Gynecol Cancer 23: 264-267.

*Correspondence: Dr S Bendifallah; E-mail: sofiane.bendifallah@yahoo.fr

Published online 22 April 2014

(C) 2014 Cancer Research UK. All rights reserved 0007-0920/14

\section{(C)(1)(0)}

http://creativecommons.org/licenses/by-nc-sa/3.0/

\title{
Comment on 'Existing prognostic models, but not neutrophil-to-lymphocyte ratio, are prognostic in malignant mesothelioma'
}

\author{
S C-H Kao ${ }^{* 1,2}$, N van Zandwijk ${ }^{1,3}$ and S Clarke ${ }^{3,4}$
}

${ }^{1}$ Asbestos Diseases Research Institute, Sydney, New South Wales, Australia; ${ }^{2}$ Department of Medical Oncology, Chris O'Brien Lifehouse, Sydney, New South Wales, Australia; ${ }^{3}$ Faculty of Medicine, University of Sydney, Sydney, New South Wales, Australia and ${ }^{4}$ Department of Medical Oncology, Royal North Shore Hospital, Sydney, New South Wales, Australia

Sir,

We feel compelled to comment on the article of Meniawy et al (2013) to provide perspective on the value of the neutrophil to lymphocyte ratio (NLR) as a prognostic indicator in patients with malignant pleural mesothelioma (MPM). The Western Australia-based authors of this article have concluded from their analysis that the NLR did not provide prognostic value, whereas the Cancer and Leukemia Group B (CALGB) and European Organisation for Research and Treatment of Cancer (EORTC) prognostic guides did.

However, there are some flaws in the data that have not been adequately acknowledged and that might have had a major impact on the conclusions. The principal flaw was that although intended to be an analysis of 369 consecutive patients presenting to a single treatment centre, this number was reduced by 95 (26\%) based on failure to meet fairly arbitrarily defined inclusion criteria of: availability of a full blood count within 90 days of diagnosis; cytologically or histologically confirmed diagnosis of MPM; absence of concurrent haematological malignancy and duration of follow-up $>90$ days. A majority of patients (64) were excluded on the basis of missing laboratory data (unspecified as to which). There was no attempt to compare the characteristics of those excluded with those included to determine comparability of populations. In addition, of the remaining 274 patients, 169 (46\% of initial) were treated with chemotherapy, whereas 105 (28\%) received no systemic chemotherapy. In spite of $28 \%$ of patients receiving no treatment at all, the median survival for the entire group was 13.3 months with a median of 15.3 months for the chemotherapy group. These data appear to show unusually good overall survivals and are suggestive of selection bias, possibly caused by the exclusion of the 95 patients. In our original study in consecutive patients receiving systemic chemotherapy for MPM (Kao et al, 2010), the median survival was very similar to that reported by Vogelzang et al (2003) in their phase III study that compared pemetrexed and cisplatin with cisplatin alone.

The findings of Meniawy and colleagues are also contradictory to the findings of other investigators in regard to the prognostic significance of NLR in MPM and numerous investigators in other tumour types (Cedres et al, 2012, 2013; Pinato et al, 2012; Guthrie et al, 2013; Paramanathan et al, 2014); however, the contradictory nature of their own findings was not adequately highlighted or explanation attempted. We have recently presented the outcomes of prognostic factors in a large cohort of patients $(n=913)$ based on the clinical and laboratory data extracted from the records of the Dust Diseases Board of New South Wales (NSW), where median survival of patients was 10 months (Linton et al, 2013). In this large population-based study including $>90 \%$ of the NSW patients seeking compensation from 2002 to 2009 , NLR $>5$ was again found to be an independent poor prognostic factor $(\mathrm{HR}=1.21 ; \mathrm{CI}$ : $1.02-1.44 ; P=0.03)$ in multivariate analysis (624 patients in the model), along with non-epithelial histology, age $>70$ years, male gender, stage III/IV, platelet count $\geqslant 400$, haemoglobin $>1 \mathrm{~g} \mathrm{dl}^{-1}$ pemetrexed chemotherapy and not receiving extrapleural pneumonectomy (EPP). Although the clinical factors were not in the final multivariate model, performance status was indirectly assessed in the model by including patients who received chemotherapy and EPP. decrease, negative calretinin staining in tumour specimen, not receiving

In addition, we felt that the interesting observation of the significant predictive value of normalisation of NLR after one cycle of chemotherapy was brushed over in the article. This confirmatory finding after our initial article (Kao et al, 2010), along with the recent study demonstrating normalisation of NLR $(<5)$ predicting for a survival benefit of 7 months in a series of 118 patients participating in phase I trials (Pinato et al, 2014), suggests that prospective validation of NLR is warranted.

Finally, there appears to be a misconception that we were seeking a universal prognostic marker that could guide treatment outcomes for all. The series investigated by us confirm that determination of the NLR is a relatively simple way to assess prognosis in certain groups of patients with MPM; however, (ongoing) prospective validation will teach us how to properly use this parameter in clinical practice.

\section{REFERENCES}

Cedres S, Montero M, Martinez P, Martinez A, Rodriguez-Freixinos V, Torrejon D, Gabaldon A, Salcedo M, Ramon Y, Cajal S, Felip E (2012) Exploratory analysis of activation of PTEN-PI3K pathway and downstream proteins in malignant pleural mesothelioma (MPM). Lung Cancer 77: 192-198.

Cedres S, Montero M, Zamora E, Martinez A, Martinez P, Farinas L, Navarro A, Torrejon D, Gabaldon A, Ramon Y, Cajal S, Felip E (2013) Expression of Wilms tumor gene (WT1) is associated with survival in malignant pleural mesothelioma. Clin Transl Oncol; e-pub ahead of print 10 December 2013; doi:10.1007/s12094-013-1146-6.

Guthrie G, Charles K, Roxburgh C, Horgan P, McMillan D, Clarke S (2013) The systemic inflammation-based neutrophil-lymphocyte ratio: experience in patients with cancer. CritRev Oncol Hematol 88: 219-230.

Kao S, Pavlakis N, Harvie R, Vardy J, Boyer M, van Zandwijk N, Clarke S (2010) High blood neutrophil-to-lymphocyte ratio is an indicator of poor prognosis in malignant mesothelioma patients undergoing systemic therapy. Clin Cancer Res 16: 5805-5813.

Linton A, Pavlakis N, Kao S, Clarke S, Vardy J, van Zandwijk N (2013) Disease an patient characteristics related to survival in a large population-based cohort of patients with malignant pleural mesothelioma (MPM). J Thorac Oncol 8(S2): S311-S312.

Meniawy T, Creaney J, Lake R, Nowak A (2013) Existing models, but not neutrophil-to-lymphocyte ratio, are prognostic in malignant mesothelioma. $\mathrm{Br}$ J Cancer 109: 1813-1820.

Paramanathan A, Saxena A, Morris D (2014) A systematic review and meta-analysis on the impact of pre-operative neutrophil lymphocyte ratio on long term outcomes after curative intent resection of solid tumours. Surg Oncol 23(1): 31-39.

Pinato D, Mauri F, Ramakrishnan R, Wahab L, Lloyd T, Sharma R (2012) Inflammation-based prognostic indices in malignant pleural mesothelioma. I Thorac Oncol 7: 587-594.

Pinato D, Stavraka C, Flynn M, Forster M, O'Cathail S, Seckl M, Kristeleit R, Olmos D, Turnbull S, Blagden S (2014) An inflammation based score can optimize the selection of patients with advanced cancer considered for early phase clinical trials. PLoS One 9(1): e83279.

Vogelzang NJ, Rusthoven JJ, Symanowski J, Denham C, Kaukel E, Ruffie P, Gatzemeier U, Boyer M, Emri S, Manegold C, Niyikiza C, Paoletti P (2003) Phase III study of pemetrexed in combination with cisplatin versus cisplatin alone in patients with malignant pleural mesothelioma. J Clin Oncol 21 : $2636-2644$

*Correspondence: Dr SC-H Kao; E-mail: steven.kao@lh.org.au Published online 8 April 2014

(c) 2014 Cancer Research UK. All rights reserved 0007-0920/14 http://creativecommons.org/licenses/by-nc-sa/3.0/

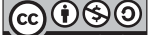

